\title{
Systematic Review of Publications Regarding Quadriceps Tendon Autograft Use in Anterior Cruciate Ligament Reconstruction
}

\author{
Walker M. Heffron, B.S., Jennifer L. Hunnicutt, Ph.D., A.T.C., John W. Xerogeanes, M.D., \\ Shane K. Woolf, M.D., and Harris S. Slone, M.D.
}

\begin{abstract}
Purpose: To perform a systematic review with quantitative and qualitative analysis of publications to date focusing on the use of quadriceps tendon (QT) autografts in anterior cruciate ligament (ACL) reconstruction and to define the regional variability, type of publication, level of evidence, journal of publication, and type of QT graft used. Methods: The PubMed, Scopus, and Google Scholar databases were systematically queried for journal articles relating to QT autografts used for ACL reconstruction through 2018. These publications were filtered for relevance and then analyzed and differentiated by publication characteristics. Results: Most articles were published in the United States and Europe, and most articles evaluating clinical outcomes were Level of Evidence III. Over $60 \%$ of the publications (115 of 187) focusing on QT for ACL reconstruction were published within the past 10 years, and 30\% (56 of 187) were published within the past 3 years. The results not only showed a recent increase in the number of publications regarding QT as a choice for the autograft harvest site in ACL reconstruction over time, but also yielded informative data regarding the publication journal, country or region, and level of evidence. Conclusions: This evaluation shows the increasing interest in the scientific evaluation of QT as a source of autograft tissue for ACL reconstruction. Clinical Relevance: Increased production of high-quality research will allow surgeons to feel more confident in their use of the QT as an autograft option in ACL reconstruction.
\end{abstract}

A nterior cruciate ligament (ACL) tears are one of the most common orthopaedic injuries seen in the United States, especially in young active individuals. ${ }^{1}$ In the United States, approximately 200,000 ACL reconstructions are performed per year. ${ }^{2}$ Since the first described ACL reconstruction in the early 20th century, the amount of research into the pathology of ACL injuries and need for surgical reconstruction has grown

From the College of Medicine, Medical University of South Carolina (W.M.H.), Charleston, South Carolina; Department of Orthopaedics, Emory University (J.L.H., J.W.X.), Atlanta, Georgia; and Department of Orthopaedic Surgery, Medical University of South Carolina (S.K.W., H.S.S.), Charleston, South Carolina, U.S.A.

The authors report that they have no conflicts of interest in the authorship and publication of this article. Full ICMJE author disclosure forms are available for this article online, as supplementary material.

Received September 10, 2018; accepted September 6, 2019.

Address correspondence to Walker M. Heffron, Medical University of South Carolina, 171 Ashley Ave, Charleston, SC 29425,U.S.A. E-mail: heffron@ musc.edu

(C) 2019 by the Arthroscopy Association of North America. Published by Elsevier Inc. This is an open access article under the CC BY-NC-ND license (http://creativecommons.org/licenses/by-nc-nd/4.0/).

2666-061X/181118

https://doi.org/10.1016/j.asmr.2019.09.001 drastically, especially over the past half century. ${ }^{3}$ As the understanding of ACL anatomy and biomechanics has improved over time, new surgical techniques have been introduced and refined.

Optimal graft type remains one of the most important and most debated surgical decisions in ACL reconstruction. In the late 1970s and early 1980s, use of bone-patellar tendon-bone graft gained popularity and is still considered the gold standard with which other grafts are compared. Since that time, hamstring grafts have gained popularity as an alternative option, largely owing to morbidity associated with patellar tendon autografts. ${ }^{3}$ Although patellar tendon and hamstring grafts remain widely used today, another alternative graft choice for ACL reconstruction has been gaining in popularity, the quadriceps tendon (QT). A technique for use of the QT as an autograft donor site for ACL reconstruction was introduced in 1979 by Marshall et al. ${ }^{4}$ Since its introduction, the QT has gained popularity for use as a graft source because of favorable biomechanics, low donor-site morbidity, large cross-sectional area of the graft, predictability, ease of harvest, and graft versatility. ${ }^{5,6}$ 

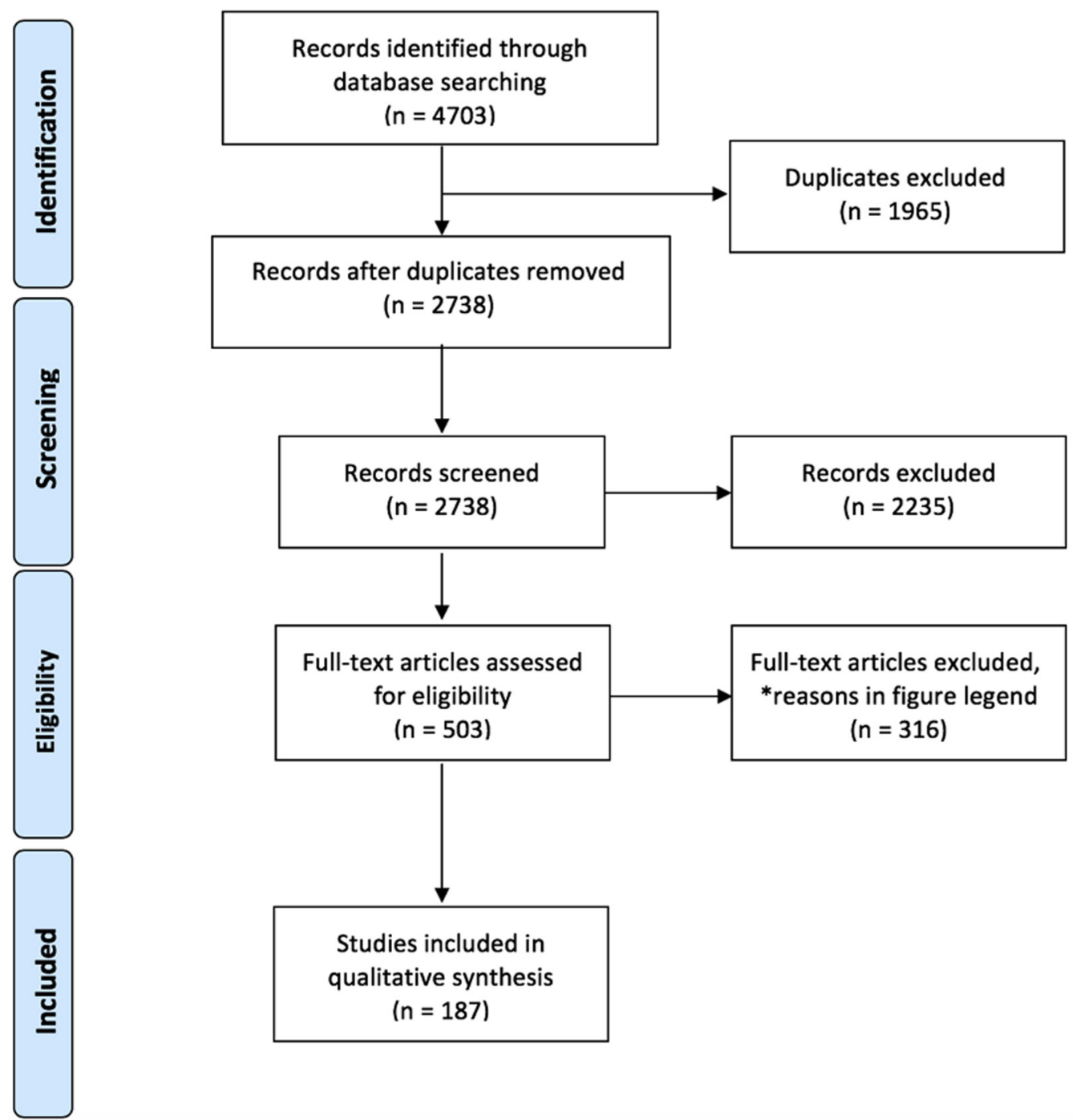

Fig 1. Preferred Reporting Items for Systematic Reviews and Meta-analyses flow diagram showing publication identification and screening process for inclusion. Publications were excluded (asterisk) if they were editorial commentaries, evaluated quadriceps tendon (QT) allografts or QT grafts for non-anterior cruciate ligament reconstruction, evaluated animal models, or failed to identify the proportion of QT grafts when multiple grafts were used.

Because there is no consensus on the optimal graft choice for ACL reconstruction, this continues to be a prevalent topic of research, often focusing on the more popular patellar tendon or hamstring grafts. However, as the use of QT in ACL reconstruction increases, so does its interest as a topic of scientific investigation. In an effort to evaluate the increasing popularity of QT autografts, the purpose of this study was to perform a systematic review with quantitative and qualitative analysis of publications to date focusing on the use of QT autografts in ACL reconstruction. In addition to evaluating the number of publications regarding QT ACL reconstruction over time, we sought to define the regional variability, type of publication, level of evidence, journal of publication, and type of QT graft used.
The hypothesis of this study was that a systematic review of ACL literature would show increasing scientific interest in the QT as a source for autograft ACL reconstruction.

\section{Methods}

The PubMed and Scopus databases were queried for journal articles focused on QT autografts being used for ACL reconstruction published through December 31, 2018. The search was performed using the following queries: (1) (quadriceps tendon[All Fields]) AND (anterior cruciate[All Fields]) and (2) (quadriceps tendon[All Fields]) AND (ACL[All Fields]). Google Scholar and the references of the articles found were reviewed to identify any articles not included in the 
Number of Publications Per Year

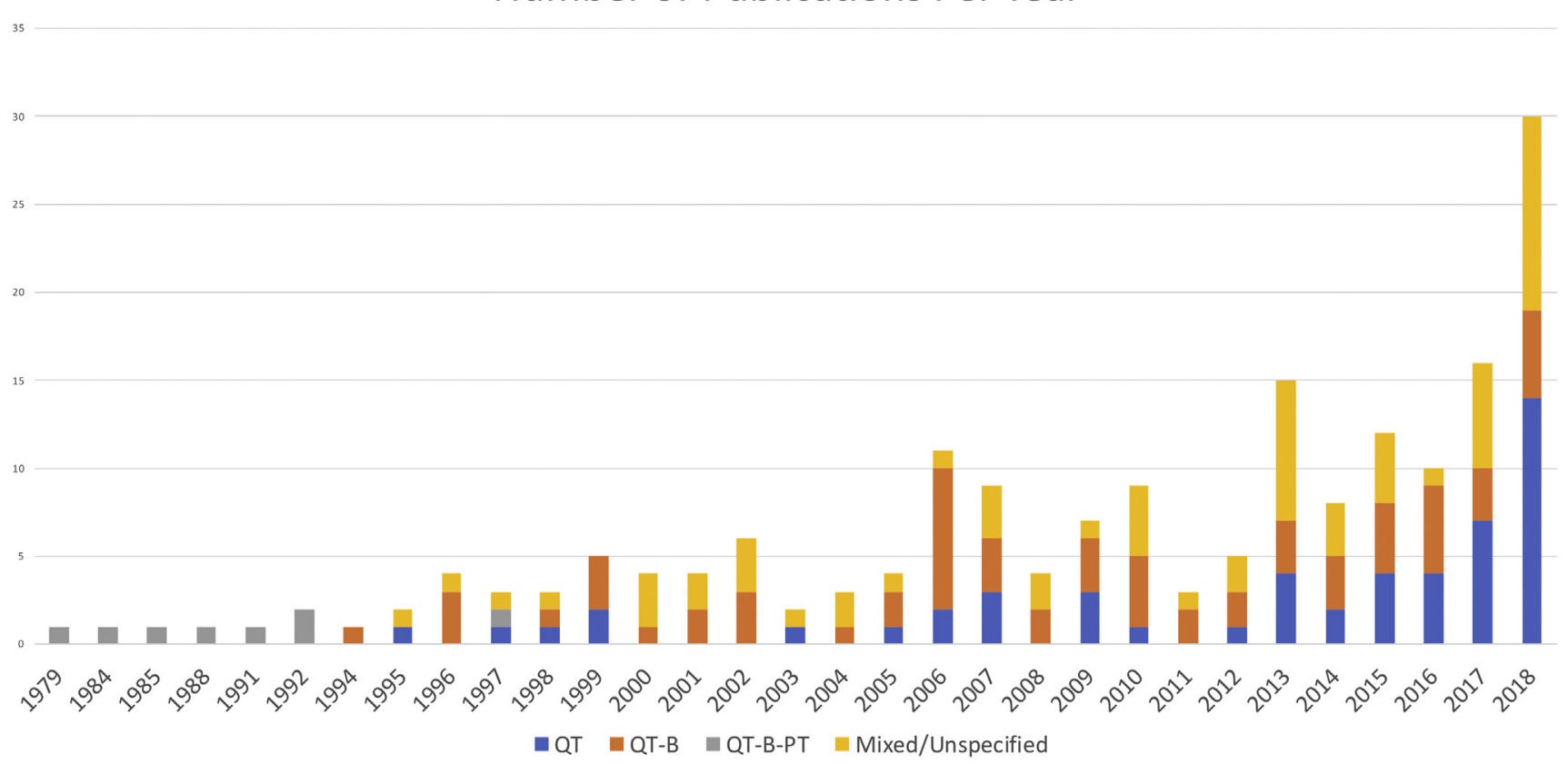

Fig 2. Number of publications involving the evaluation or use of quadriceps tendon autografts for reconstruction of the anterior cruciate ligament per year. The color bars represent the type of quadriceps tendon graft discussed in the publication: quadriceps tendon or central quadriceps tendon (QT), quadriceps tendon-bone (QT-B) (or a combination of QT and QT-B), or quadriceps tendon-bone-patellar tendon (QT-B-PT). The data represent the publication count through December 31, 2018.

primary search. These publications were then analyzed and differentiated by the following publication characteristics: year of publication, QT graft type evaluated (QT or central QT, quadriceps tendon-bone [QT-B], or quadriceps tendon-bone-patellar tendon [QT-B-PT]), journal of publication, country of the corresponding author's affiliation, and geographic region of the corresponding author's affiliation. The publications evaluating clinical outcomes were also analyzed for the level of evidence according to Oxford Centre for Evidence-Based Medicine guidelines. ${ }^{7}$ The Preferred Reporting Items for Systematic Reviews and Metaanalyses (PRISMA) guidelines were followed throughout the analyses. ${ }^{8}$

Articles discussing the use of QT autograft for primary and revision ACL reconstruction and/or biomechanical evaluation of QT grafts were included. Clinical studies that did not exclusively evaluate the QT graft were included if greater than $25 \%$ of the evaluated ACL reconstructions used QT grafts. If a publication evaluated the use of more than 1 graft type (e.g., a portion of patients received QT grafts and a portion received QTB-PT grafts) or did not specify the QT graft type, the publication was counted in the mixed/unspecified category. Studies were excluded if they evaluated QT allografts, evaluated QT grafts for non-ACL reconstruction, were conducted in animal models, or failed to identify the proportion of QT grafts when multiple grafts were used. Editorial commentaries and book chapters were excluded.

\section{Results}

Search queries 1 and 2 collectively yielded 1,179 results from PubMed and 3,524 results from Scopus, for a total of 4,703 results. The results were cross-referenced for duplicates, yielding 2,738 unique publications. After initial screening, 503 publications were assessed for eligibility. A Google Scholar search was then conducted, which revealed that no relevant articles were missed by the initial queries. The 503 full-text articles were filtered based on our inclusion and exclusion criteria, which yielded 187 publications to be included in the study (Fig 1). Of the publications focusing on QT for ACL reconstruction, $62 \%$ (115 of 187) were published within the past 10 years and $30 \%$ (56 of 187) were published within the past 3 years (Fig 2). Use of the QTB-PT graft was common in early studies when QT was first introduced as an alternative graft. Of the 25 studies published in the first 2 decades since the introductory article of Marshall et al. ${ }^{4}$ in $1979,32 \%$ (8 of 25) evaluated the QT as part of a QT-B-PT graft (Fig 2). However, in the 162 studies published since then $(87 \%$ of the total publications), QT grafts were evaluated as either QT-B grafts or QT or central QT grafts (Fig 2). In total, $96 \%$ of publications (179 of 187) evaluated QT grafts, QT-B grafts, or a combination of QT and QT-B 


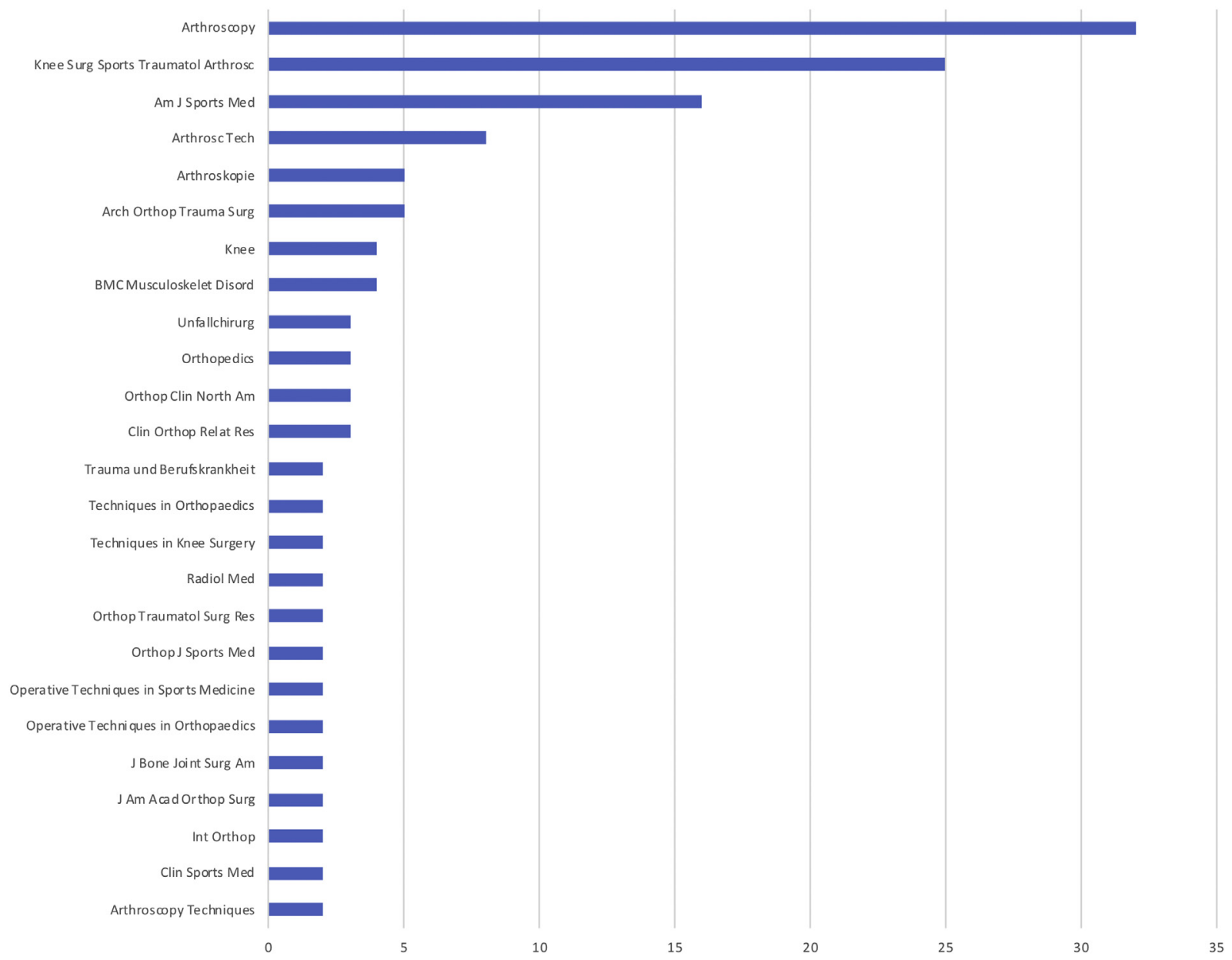

Fig 3. Number of publications per journal regarding the evaluation or use of quadriceps tendon autografts for anterior cruciate ligament reconstruction. Journals shown contributed 2 or more publications.

grafts. Most studies focusing on QT graft for ACL reconstruction were published in arthroscopy-focused or sports medicine-focused journals (Fig 3). The United States was the country with the most publications focusing on QT grafts for ACL reconstruction (Fig 4). However, when publications were evaluated by region, Europe was responsible for the majority (Fig 5). Most articles evaluated clinical outcomes $(\mathrm{n}=112$, $57 \%$ ). Of the clinical outcome studies, $72 \%$ were Level of Evidence III (Fig 6). Of the 16 Level of Evidence I or II publications, 11 were published within the past 5 years.

\section{Discussion}

Through a quantitative and qualitative evaluation of the current literature, this study shows the increasing scientific interest in the QT as a source of autograft tissue for use in ACL reconstruction since its introduction in 1979. The vast majority of studies have been published within the past decade, and higher-quality studies including prospective and randomized controlled trials are a product of recent investigation. Although most publications were published in sports medicine and arthroscopic journals based in the United States and Europe, it is evident that the interest in QT use in ACL reconstruction expands far beyond these regions.

As the pressure continues to mount for physicians to practice evidence-based medicine, ${ }^{9}$ it is imperative that graft choice for ACL reconstruction be based on quality clinical research. This evaluation shows increasing interest in research on QT as an alternative graft source. This may have led surgeons to increased use of QT grafts for ACL reconstructions, as reported in recent literature. In a 2010 review, van Eck et al. ${ }^{10}$ found that only $2.5 \%$ of all ACL reconstructions used QT. In 2014, Middleton et al. ${ }^{11}$ found QTs being used in $11 \%$ of ACL reconstructions. In addition, our study shows a proportionate shift in the use of central QT grafts versus QT-B and QT-B-PT grafts. This is possibly related to improvement and expansion of the available fixation techniques, which enhances the usability and reliability 
Number of Publications by Country

$$
70
$$

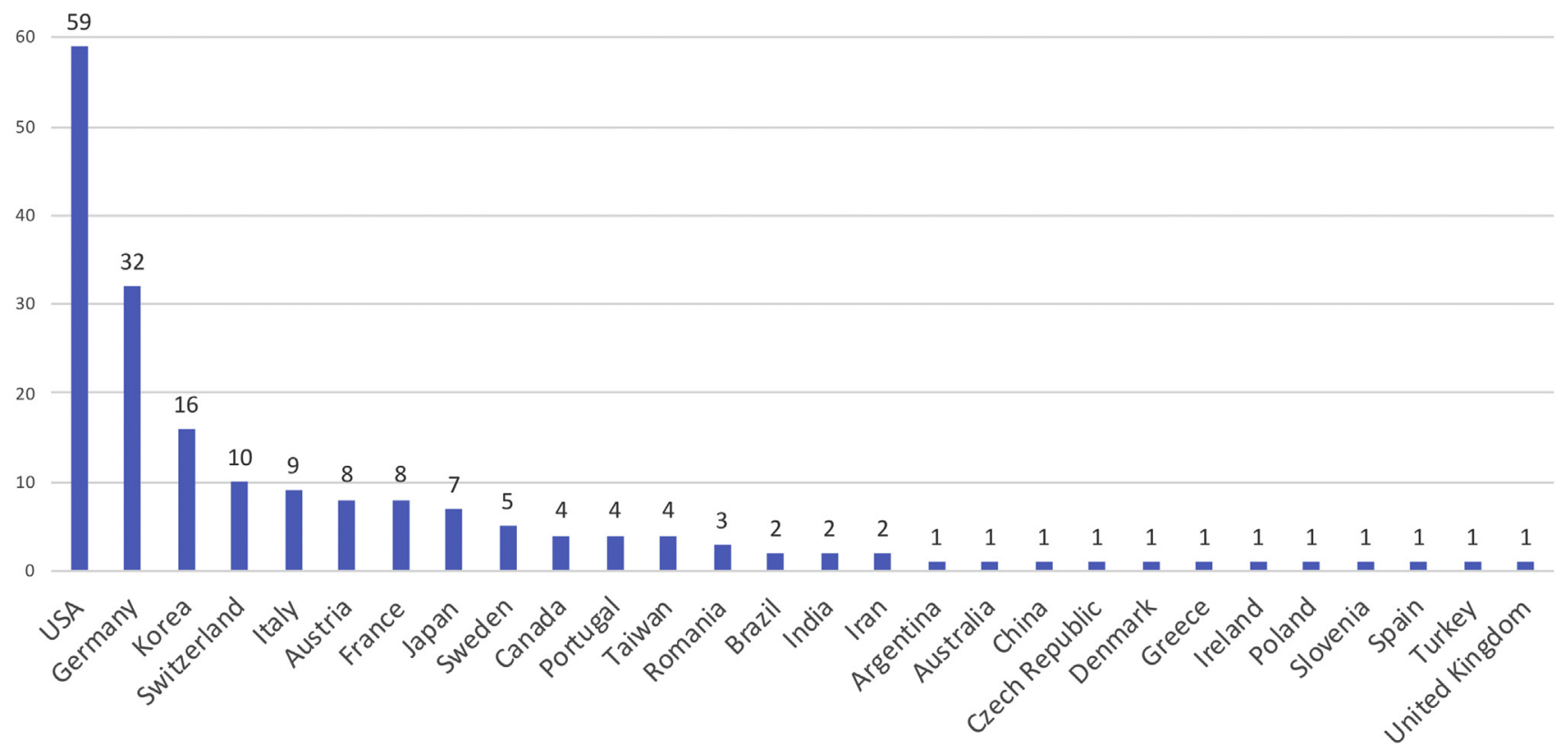

Fig 4. Number of publications per country. The country of origin was considered the location of the corresponding author's affiliation.

of an all-soft tissue cylindrical unlooped graft such as the QT graft. The results of our study show increased interest in critical evaluation regarding the use of the QT autograft for ACL reconstruction and substantiate the need for research studies of higher levels of evidence, including randomized controlled trials, comparing the QT with other autograft choices.
Over the past half century, increased research productivity into ACL pathology, surgical techniques, and graft choices has improved the evidence-based practice regarding the treatment of ACL pathology. Many proposed advantages to using the QT for ACL reconstruction have been discussed in the literature. Several studies have evaluated the anatomic qualities of the

Fig 5. Number of publications by region. The region was assigned based on the geographical location of the corresponding author's affiliation.

Number of Publications by Region

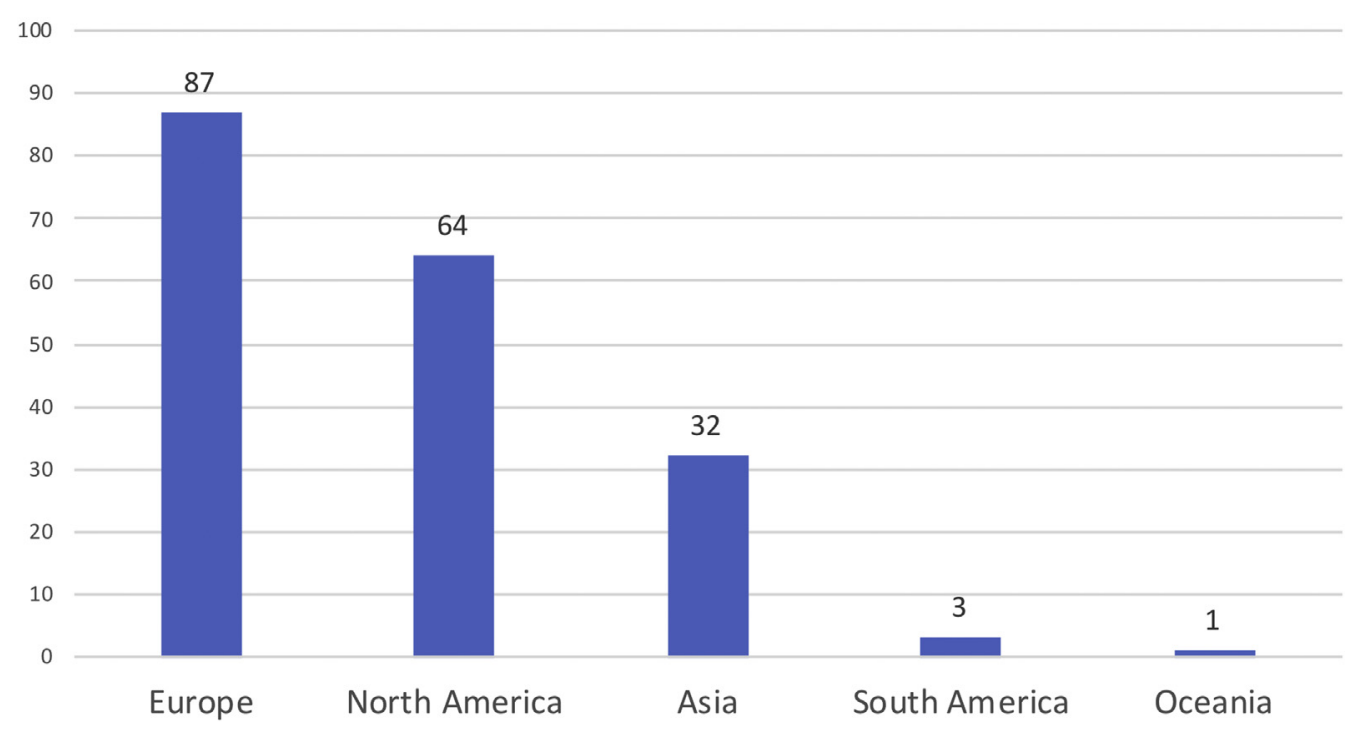




\section{Level of Evidence for Publications Evaluating Clinical Outcome}

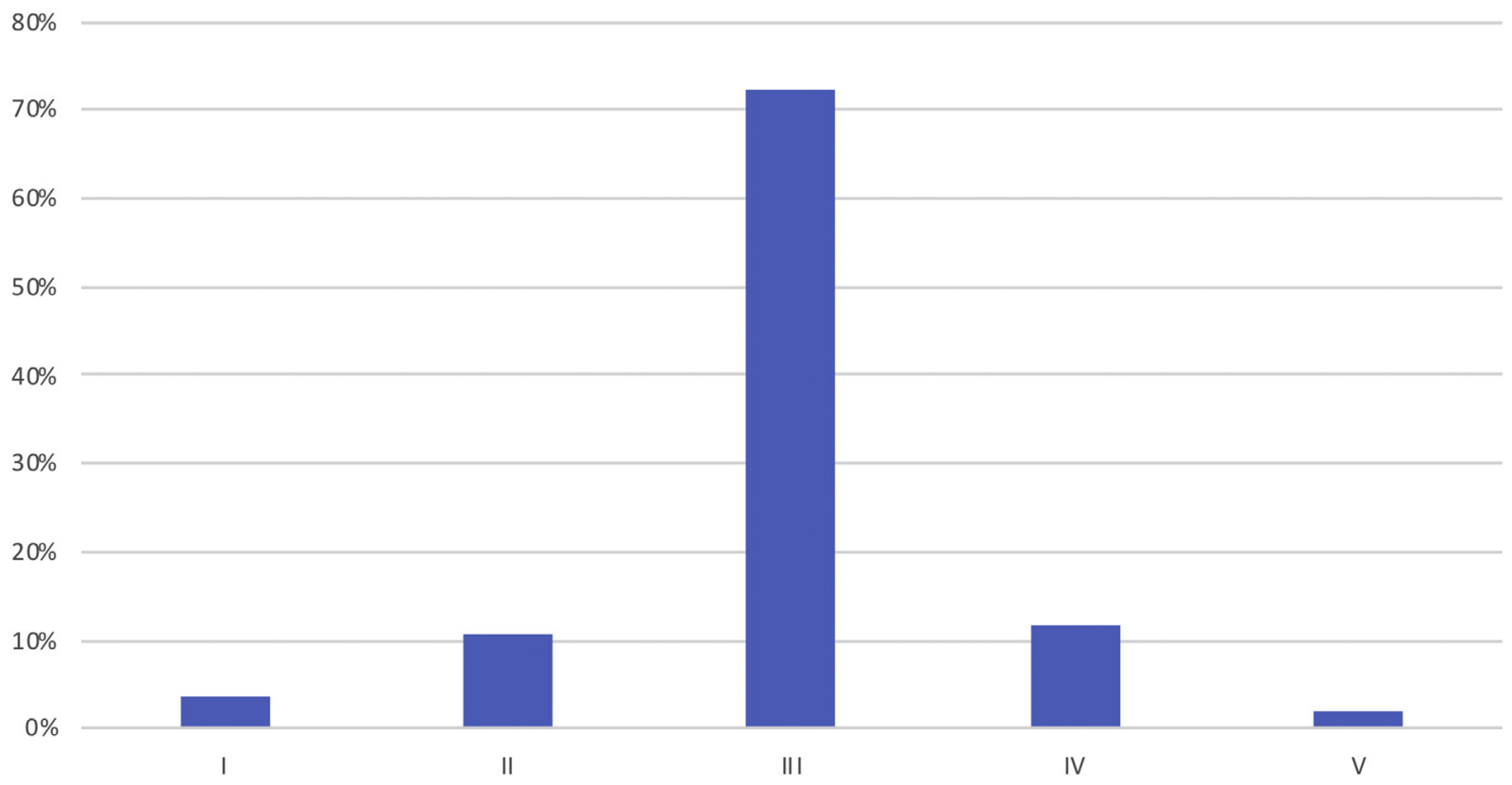

Fig 6. Level of evidence of clinical publications. Publications that evaluated clinical outcomes (112 of 197) were sorted based on level of evidence (using Oxford Centre for Evidence-Based Medicine guidelines ${ }^{7}$ ). Values are expressed as a percentage of the clinical publications $(\mathrm{n}=112)$.

graft tissue and revealed that the QT has a greater anatomic area for graft harvesting versus patellar tendon grafts. ${ }^{12,13}$ Harris et al. ${ }^{14}$ found that greater collagen levels in the QT yielded more favorable biomechanical properties versus patellar tendon grafts of comparable width. Adams et al. ${ }^{15}$ showed that the post-harvest strength of the QT was greater than the strength of the intact patellar tendon. Several studies have associated small autograft size with increased failure rates, especially with graft diameters smaller than $8 \mathrm{~mm} .{ }^{16,17}$ Thus, a potential surgical advantage to the QT is that it has a larger and more favorable area from which to harvest the graft. ${ }^{18}$

Multiple recently published systematic reviews have supported the use of the QT as a viable alternative graft option based on clinical and functional outcomes after ACL reconstruction. ${ }^{6,19,20}$ Several recent studies have directly compared the clinical outcomes of QT grafts versus quadrupled hamstring grafts, showing equivalent or superior functional outcomes and less postoperative donor-site pain with QT grafts. ${ }^{19,21,22}$

This systematic review shows that since the introduction of the QT autograft by Marshall et al. ${ }^{4}$ in 1979 , research regarding its use has been increasing steadily. This increase in research reflects the growth in popularity as an alternative choice for autograft tissue in ACL reconstruction. The strengths of this study include the comprehensive search of the literature through the PubMed, Scopus, and Google Scholar databases. Use of these established, public databases gives this study reproducibility and ensures the quality of the included publications. Thus, this evaluation gives an adequate snapshot, through a controlled window, of the state of research into QT autograft use for ACL reconstruction. Future work will include a further systematic literature review and meta-analysis on the research using QT autograft once research studies with higher levels of evidence are conducted and disseminated.

\section{Limitations}

This study is not without limitations. Although it does show the increasing volume of published evidence to support QT use in ACL reconstruction, it does not attempt to evaluate or compare the clinical outcomes of QT grafts versus alternative graft sources. In addition, although a rigorous systematic search of the literature was performed, it is possible that not all relevant articles were included in this review.

\section{Conclusions}

This evaluation shows the increasing interest in the scientific evaluation of QT as a source of autograft tissue for ACL reconstruction.

\section{References}

1. Sanders TL, Maradit Kremers H, Bryan AJ, et al. Incidence of anterior cruciate ligament tears and reconstruction: A 
21 -year population-based study. Am J Sports Med 2016;44: 1502-1507.

2. Gottlob CA, Baker CL Jr, Pellissier JM, Colvin L. Cost effectiveness of anterior cruciate ligament reconstruction in young adults. Clin Orthop Relat Res 1999:272-282.

3. Chambat P, Guier C, Sonnery-Cottet B, Fayard JM, Thaunat M. The evolution of ACL reconstruction over the last fifty years. Int Orthop 2013;37:181-186.

4. Marshall JL, Warren RF, Wickiewicz TL, Reider B. The anterior cruciate ligament: A technique of repair and reconstruction. Clin Orthop Relat Res 1979:97-106.

5. Chen $\mathrm{CH}$, Chen WJ, Shih CH. Arthroscopic anterior cruciate ligament reconstruction with quadriceps tendonpatellar bone autograft. J Trauma 1999;46:678-682.

6. Slone HS, Romine SE, Premkumar A, Xerogeanes JW. Quadriceps tendon autograft for anterior cruciate ligament reconstruction: A comprehensive review of current literature and systematic review of clinical results. Arthroscopy 2015:31:541-554.

7. OCEBM Levels of Evidence Working Group. The Oxford 2011 levels of evidence. Oxford Centre for Evidence-Based Medicine 2018. Published, http://www.cebm.net/index. aspx?o=5653. Accessed July 8, 2018.

8. Liberati A, Altman DG, Tetzlaff J, et al. The PRISMA statement for reporting systematic reviews and metaanalyses of studies that evaluate health care interventions: Explanation and elaboration. J Clin Epidemiol 2009;62:el-e34.

9. Masic I, Miokovic M, Muhamedagic B. Evidence based medicine-New approaches and challenges. Acta Inform Med 2008;16:219-225.

10. van Eck CF, Schreiber VM, Mejia HA, et al. "Anatomic" anterior cruciate ligament reconstruction: A systematic review of surgical techniques and reporting of surgical data. Arthroscopy 2010;26:S2-S12.

11. Middleton KK, Hamilton T, Irrgang JJ, Karlsson J, Harner CD, Fu FH. Anatomic anterior cruciate ligament (ACL) reconstruction: A global perspective. Part 1. Knee Surg Sports Traumatol Arthrosc 2014;22:1467-1482.

12. Fulkerson JP, Langeland R. An alternative cruciate reconstruction graft: The central quadriceps tendon. Arthroscopy 1995;11:252-254.

13. Xerogeanes JW, Mitchell PM, Karasev PA, Kolesov IA, Romine SE. Anatomic and morphological evaluation of the quadriceps tendon using 3-dimensional magnetic resonance imaging reconstruction: Applications for anterior cruciate ligament autograft choice and procurement. Am J Sports Med 2013;41:2392-2399.

14. Harris NL, Smith DA, Lamoreaux L, Purnell M. Central quadriceps tendon for anterior cruciate ligament reconstruction. Part I: Morphometric and biomechanical evaluation. Am J Sports Med 1997;25:23-28.

15. Adams DJ, Mazzocca AD, Fulkerson JP. Residual strength of the quadriceps versus patellar tendon after harvesting a central free tendon graft. Arthroscopy 2006;22:76-79.

16. Conte EJ, Hyatt AE, Gatt CJ Jr, Dhawan A. Hamstring autograft size can be predicted and is a potential risk factor for anterior cruciate ligament reconstruction failure. Arthroscopy 2014;30:882-890.

17. Magnussen RA, Lawrence JT, West RL, Toth AP, Taylor DC, Garrett WE. Graft size and patient age are predictors of early revision after anterior cruciate ligament reconstruction with hamstring autograft. Arthroscopy 2012;28:526-531.

18. Slone HS, Ashford WB, Xerogeanes JW. Minimally invasive quadriceps tendon harvest and graft preparation for all-inside anterior cruciate ligament reconstruction. Arthrosc Tech 2016;5:e1049-e1056.

19. Mouarbes D, Menetrey J, Marot V, Courtot L, Berard E, Cavaignac E. Anterior cruciate ligament reconstruction: A systematic review and meta-analysis of outcomes for quadriceps tendon autograft versus bone-patellar tendon-bone and hamstring-tendon autografts. Am J Sports Med 2019:363546518825340.

20. Mulford JS, Hutchinson SE, Hang JR. Outcomes for primary anterior cruciate reconstruction with the quadriceps autograft: A systematic review. Knee Surg Sports Traumatol Arthrosc 2013;21:1882-1888.

21. Buescu CT, Onutu AH, Lucaciu DO, Todor A. Pain level after ACL reconstruction: A comparative study between free quadriceps tendon and hamstring tendons autografts. Acta Orthop Traumatol Turc 2017;51:100-103.

22. Runer A, Wierer G, Herbst E, et al. There is no difference between quadriceps- and hamstring tendon autografts in primary anterior cruciate ligament reconstruction: A 2-year patient-reported outcome study. Knee Surg Sports Traumatol Arthrosc 2017;26:605-614. 